\title{
Perdas de nitrogênio por volatilização de amônia e resposta do arroz irrigado à aplicação de ureia tratada com o inibidor de urease NBPT
}

\author{
Nitrogen losses by ammonia volatilization and lowland rice response to NBPT urease \\ inhibitor-treated urea
}

\author{
Walkyria Bueno ScivittaroI ${ }^{\text {Daiana Ribeiro Nunes Gonçalves }}{ }^{\text {II }}$ Marcos Lima Campos do Vale $^{\text {II }}$ \\ Vanessa Gentil Ricordi ${ }^{\text {III }}$
}

\section{RESUMO}

A ureia é a principal fonte de nitrogênio $(N)$ para o arroz irrigado, porém é bastante suscetível a perdas por volatilização de amônia. O uso do inibidor de urease $\mathrm{N}$ - $(\mathrm{n}$ butil) triamida tiofosfórica (NBPT) pode minimizar esse problema, inibindo temporariamente a degradação enzimática da ureia. O objetivo deste trabalho foi avaliar, em duas condições de umidade do solo, o efeito do tratamento da ureia com o inibidor de urease NBPT sobre as perdas de $N$ por volatilização de amônia e seu reflexo na produtividade de grãos e acumulação de nitrogênio pelo arroz irrigado (Oryza sativa). O experimento foi realizado em um Planossolo Háplico, em Capão do Leão, Rio Grande do Sul (RS). Avaliaram-se as combinações de duas condições de umidade do solo por ocasião da primeira aplicação de $\mathrm{N}$ em cobertura, no início do perfilhamento (solo úmido e saturado), duas fontes de nitrogênio (ureia não tratada e ureia tratada com NBPT) e três intervalos de tempo entre a primeira aplicação de $N$ em cobertura e o início da irrigação (um, cinco e 10 dias). A dose utilizada de $N$ foi de $120 \mathrm{~kg} \mathrm{ha}^{-1}$, parcelados $90 \mathrm{~kg} \mathrm{ha}^{-1}$ no início do perfilhamento, de acordo com os tratamentos, e $30 \mathrm{~kg} \mathrm{ha}^{-1}$, na diferenciação da panícula. Os tratamentos foram dispostos em delineamento de blocos ao acaso, com quatro repetições. As perdas de $N$ por volatilização de amônia decorrentes do uso de ureia variaram de 15 (solo saturado) a 22\% (solo úmido) do nitrogênio aplicado, quando o intervalo entre a aplicação desse fertilizante e o início da irrigação foi de 10 dias. Estas foram reduzidas em 83 e $88 \%$ em solo saturado e úmido, respectivamente, com a adição de ureia com NBPT. Apenas na aplicação de $N$ realizada 10 dias antes do início da irrigação o uso de NBPT proporcionou maiores produtividade de grãos e acumulação de nitrogênio pelo arroz. O tratamento da ureia com NBPT possibilita que sua aplicação anteceda em até 10 dias o início da irrigação, sem prejuízos para a produtividade de grãos e a acumulação de nitrogênio pelo arroz.

Palavras-chave: adubo nitrogenado, ureia, eficiência do fertilizante, volatilização de amônia, $N$ - $(n-$ butil) triamida tiofosfórica.

\section{ABSTRACT}

Urea is the main nitrogen source used in flooded rice fields, but it is prone to ammonia volatilization losses. The use of $\mathrm{N}$-(n-butyl) thiophosphoric triamide (NBPT) urease inhibitor, as urea additive, can reduce this inconvenient, inhibiting temporarily urea enzymatic degradation. An experiment was carried out on a Planossolo Háplico (Albaqualf), from October 2004 to April 2005, in Capão do Leão, RS, Brazil, to evaluate the effect of NBPT inhibitor-treated urea on nitrogen losses by ammonia volatilization, rice grain yield, and rice nitrogen accumulation, in two soil moisture conditions. The treatments were arranged as a randomized complete block with two soil moisture conditions (moist and muddy), two nitrogen sources (non treated urea and urea treated with NBPT) and three time intervals between nitrogen application and flooding (1, 5, and 10 days) in a factorial design with four replications. Nitrogen rate was $120 \mathrm{~kg} \mathrm{~N} \mathrm{ha}^{-1}$, applied at 4- to 5- leaf stage (90kg $\left.\mathrm{N} \mathrm{ha}^{-1}\right)$ and at panicle

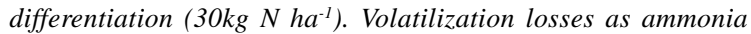
from the urea treatment varied from 15\% (muddy soil) to 22\% (moist soil) of the applied $N$, when the time interval between nitrogen application and flooding was 10 days. Addition of NBPT reduced $83 \%$ and $88 \%$ ammonia volatilization losses in muddy soil and moist soil conditions, respectively. For the 10 days interval between nitrogen application and flooding, NBPT treated urea promoted greater rice yield and nitrogen

IEmbrapa Clima Temperado, 96001-970, Pelotas, RS, Brasil. E-mail: walkyria.scivittaro@cpact.embrapa.br. Autor para correspondência.

"Departamento de Solos, Faculdade de Agronomia Eliseu Maciel (FAEM), Universidade Federal de Pelotas (UFPel), Pelotas, RS, Brasil.

II'Departamento de Química Orgânica, IQG, UFPel, Pelotas, RS, Brasil. 
accumulation, compared to non treated urea. The use of NBPT inhibitor makes possible applying urea up to ten days before flooding with no decrease on rice grain yield and nitrogen accumulation.

Key words: nitrogen fertilizer, urea, fertilizer efficiency, ammonia volatilization, $\mathrm{N}$-(n-butyl) thiophosphoric triamide.

\section{INTRODUÇÃO}

As fontes nitrogenadas amoniacais e amídicas são teoricamente mais adequadas e, portanto, recomendadas para o arroz irrigado por inundação. Dentre estas, a ureia destaca-se pelo elevado conteúdo de nitrogênio (N) e menor custo por unidade do nutriente aplicado. A despeito de tais aspectos favoráveis, esse fertilizante apresenta como característica indesejável reação inicial alcalina no solo, resultando em perdas por volatilização de amônia, quando aplicado em superfície.

Além da atividade da urease, que controla a taxa de hidrólise da ureia, a intensidade das perdas de amônia da ureia é influenciada pelas condições ambientais, pela cobertura e pelo tipo de solo, pelas práticas culturais e pelo manejo do fertilizante (dose e forma de aplicação) (ANJOS \& TEDESCO, 1976; XU et al., 1993). A interação desses fatores determina variabilidade na eficiência de utilização de $\mathrm{N}$ da ureia pelas culturas. Em arroz irrigado, as perdas de amônia podem variar de 20 a $80 \%$ da quantidade aplicada do nutriente(VENTURA \& YOSHIDA, 1977; FILLERY et al., 1984; ZHU, 1992).

A atual recomendação de adubação nitrogenada para o Sul do Brasil (CQFS-RS/SC, 2004) prevê, para o arroz cultivado no sistema de semeadura em solo seco, que a primeira aplicação de $\mathrm{N}$ em cobertura no início do perfilhamento anteceda a entrada de água na lavoura, de forma que a água de irrigação promova sua incorporação ao solo, minimizando as perdas de amônia da ureia. Entretanto, variações no manejo da cultura e na operacionalidade da lavoura condicionam intervalos de tempo variáveis entre a aplicação do fertilizante e o início da irrigação, em alguns casos superiores a cinco dias. Nesse período, a ureia depositada na superfície do solo fica exposta a perdas, que podem se refletir em redução na produtividade da cultura. Além disso, em áreas de várzea, o solo pode estar saturado por ocasião da aplicação da primeira aplicação de N em cobertura, impedindo a incorporação efetiva da ureia pela água de irrigação, favorecendo as perdas de amônia. No Brasil, LARA CABEZAS et al. (1997; 2000) e VITTI et al. (2007) determinaram, respectivamente, redução na produtividade de milho e de cana-de-açúcar decorrente da aplicação de ureia em superfície.
Em arroz irrigado, uma alternativa para situações em que não é possível entrar com a água rapidamente na lavoura após a primeira aplicação de $\mathrm{N}$ em cobertura e/ou quando a umidade do solo é elevada nessa ocasião, possibilitando a rápida hidrólise e liberação de amônia pela ureia, consiste no tratamento prévio do fertilizante com inibidor de urease, que atua sobre a molécula de ureia por um período prédeterminado, minimizando a volatilização de amônia. Recentemente, foi disponibilizada, no mercado nacional, ureia tratada com o inibidor de urease $\mathrm{N}$-(n-butil) triamida tiofosfórica (NBPT). Esse aditivo inibe a degradação enzimática da ureia por um período de até 14 dias, reduzindo as possíveis perdas de amônia (AGROTAIN, 2001), podendo representar mais uma opção de manejo do nitrogênio no arroz irrigado. Em cana-de-açúcar, a eficiência desse inibidor foi comprovada, proporcionando reduções de 15 a 78\% nas perdas por volatilização de ureia aplicada sobre a palha, dependendo das condições climáticas nos dias subsequentes à aplicação de nitrogênio (CANTARELLA et al., 2008).

Realizou-se um experimento para avaliar, sob duas condições de umidade do solo, o efeito do tratamento da ureia com o inibidor de urease NBPT sobre as perdas de $\mathrm{N}$ por volatilização de amônia e seu reflexo na produtividade de grãos e acumulação de nitrogênio pelo arroz irrigado.

\section{MATERIAL E MÉTODOS}

O estudo foi desenvolvido no ano agrícola 2004/05, na Estação Experimental Terras Baixas da Embrapa Clima Temperado, em Capão do Leão, Rio Grande do Sul (RS). O solo da área experimental é classificado como Planossolo Háplico, apresentando as seguintes características químicas, na profundidade de $0-20 \mathrm{~cm}$, por ocasião da instalação do experimento: $\mathrm{pH}_{\text {(água) }}: 5,6 ; 14 \mathrm{~g} \mathrm{dm}^{-3}$ de MO; 8,7 $\mathrm{mg} \mathrm{dm}^{-3}$ de P; 38mg $\mathrm{dm}^{-3}$ de K; $0,1 \mathrm{cmol}_{\mathrm{c}} \mathrm{dm}^{-3}$ de Al; $2,8 \mathrm{cmol}_{\mathrm{c}} \mathrm{dm}^{-3}$ de Ca; $1,4 \mathrm{cmol}_{\mathrm{c}} \mathrm{dm}^{-3}$ de $\mathrm{Mg}$ e saturação por bases de $58 \%$. Os teores de matéria orgânica, fósforo e potásssio são interpretados, respectivamente, como baixo, alto e baixo, conforme CQFS-RS/SC (2004).

Os tratamentos consistiram na combinação de duas condições de umidade do solo por ocasião da primeira aplicação de $\mathrm{N}$ em cobertura no início do perfilhamento do arroz (solo úmido, com umidade gravimétrica de aproximadamente $16 \%$, e solo saturado, com umidade gravimétrica de aproximadamente $24 \%$ ), duas fontes de nitrogênio (ureia não tratada e ureia tratada com o inibidor de urease NBPT, na concentração de $0,05 \%$ ) e três intervalos de tempo entre a primeira 
aplicação de $\mathrm{N}$ em cobertura e a entrada de água na lavoura (um, cinco e 10 dias). Estes foram dispostos em delineamento de blocos ao acaso, em esquema fatorial, com quatro repetições. O início da irrigação ocorreu simultaneamente para todos os tratamentos, aos 33 dias após a emergência das plântulas, correspondendo ao estádio V5, segundo a escala de COUNCE et al. (2000). As diferenças nos períodos entre a aplicação do N e o início da irrigação foram obtidas por meio de variações nas épocas de aplicação das fontes de nitrogênio. A condição de solo saturado foi obtida mediante a irrigação das parcelas, por inundação intermitente, com antecedência de um dia das aplicações de N. Por ocasião da primeira adubação nitrogenada em cobertura no início do perfilhamento, determinouse a umidade gravimétrica do solo, sendo coletadas quatro subamostras, na profundidade de $0-10 \mathrm{~cm}$, das parcelas relativas aos tratamentos com solo úmido e saturado.

A dose de $\mathrm{N}$ utilizada foi de $120 \mathrm{~kg} \mathrm{ha}^{-1}$, estabelecida de acordo com os resultados da análise de solo e as recomendações para a cultura, para uma expectativa de produtividade superior a $9 \mathrm{t} \mathrm{ha}^{-1}$ (CQFSRS/SC, 2004). Esta foi parcelada entre o início do perfilhamento e a diferenciação da panícula - estádio R1, conforme COUNCE et al. (2000). Na primeira época, aplicaram-se $90 \mathrm{~kg} \mathrm{ha}^{-1}$ de $\mathrm{N}$ antes da entrada de água, de acordo com os tratamentos, e na segunda, $30 \mathrm{~kg} \mathrm{ha}^{-1}$ de $\mathrm{N}$, na forma de ureia, sobre uma lâmina de água não circulante.

A semeadura da cultivar BRS 7 'Taim' foi realizada em 27/10/04, em sistema convencional de preparo de solo, sendo utilizado espaçamento entrelinhas de $17,5 \mathrm{~cm}$ e $130 \mathrm{~kg} \mathrm{ha}^{-1}$ de sementes, visando à obtenção de uma população de 200 a 300 plantas $\mathrm{m}^{-2}$ (SOSBAI, 2007). Na semeadura, aplicaramse a lanço e incorporaram-se $50 \mathrm{~kg} \mathrm{ha}^{-1}$ de $\mathrm{P}_{2} \mathrm{O}_{5}$ (superfosfato triplo) e $60 \mathrm{~kg} \mathrm{ha}^{-1}$ de $\mathrm{K}_{2} \mathrm{O}$ (cloreto de potássio). Para complementar a dose prevista de potássio, aplicaram-se, no estádio $\mathrm{R} 1,20 \mathrm{~kg} \mathrm{ha}^{-1} \mathrm{de} \mathrm{K}_{2} \mathrm{O}$, na forma de cloreto de potássio (CQFS-RS/SC, 2004). Os demais tratos culturais seguiram as indicações da pesquisa para o arroz irrigado (SOSBAI, 2007).

A avaliação das perdas de nitrogênio por volatilização de amônia da ureia tratada ou não com NBPT foi realizada a partir de coletores construídos de acordo com o modelo de NÖMMIK (1973), modificado por CANTARELLA et al. (2003). Os coletores consistiram de tubos vazados, em PVC, com 25,4cm de diâmetro interno e $50 \mathrm{~cm}$ de altura, nos quais foram dispostos dois discos de espuma de polietileno com $2 \mathrm{~cm}$ de espessura, a $2,5 \mathrm{~cm}$ e $15 \mathrm{~cm}$ da extremidade superior. Ambos os discos de espuma foram impregnados com solução de ácido fosfórico e glicerina para adsorver a amônia volatilizada, que foi extraída com solução molar de cloreto de potássio e determinada por destilação por arraste de vapor. O disco superior destinou-se à adsorção de possíveis contaminações de amônia proveniente da atmosfera e o inferior destinou-se à adsorção da amônia oriunda da ureia. A extremidade superior do tubo foi coberta com filme plástico afixado com cinta de látex e por um tampo em madeira.

As câmaras de volatilização foram instaladas nas parcelas em que a primeira cobertura com $\mathrm{N}$ antecedeu em 10 dias o início da irrigação, sendo enterradas até a profundidade de $10 \mathrm{~cm}$. As determinações de amônia volatilizada foram feitas aos dois, cinco, 10, 15 e 20 dias após a aplicação dos fertilizantes, sendo as duas últimas realizadas após o alagamento do solo, condição que, no interior das câmaras, foi estabelecida pela adição de água em quantidade suficiente para o estabelecimento de uma lâmina de $10 \mathrm{~cm}$ de espessura, à semelhança da mantida no restante da parcela experimental.

A produtividade de grãos de arroz foi determinada na maturação de colheita (estádio R9), pela coleta manual da área útil das parcelas (nove linhas de $4 \mathrm{~m}$ de comprimento). O material colhido foi trilhado, secado e amostrado para determinação da umidade, sendo convertidos os valores de produtividade para $130 \mathrm{~g} \mathrm{~kg}^{-1}$ de umidade. Realizou-se, ainda, a amostragem da parte aérea das plantas (três linhas de $0,5 \mathrm{~m}$ de comprimento) para a determinação da produção de matéria seca. Esse material vegetal foi homogeneizado e amostrado para a determinação da concentração de nitrogênio. A partir dos dados de produção de matéria seca e de concentração de $\mathrm{N}$ no tecido vegetal, determinaram-se as quantidades de $\mathrm{N}$ acumuladas nas plantas.

Os dados obtidos foram submetidos à análise de variância, sendo comparadas as médias dos fatores umidade do solo, fonte de nitrogênio e intervalo de tempo entre a primeira aplicação de $\mathrm{N}$ em cobertura e a entrada de água pelo teste de Tukey, a 5\%. As causas de variação foram duas (condição de umidade do solo e fonte de nitrogênio) exclusivamente para a variável perda acumulada de $\mathrm{N}-\mathrm{NH}_{3}$.

\section{RESULTADOS E DISCUSSÃO}

Durante o período de avaliação, as perdas de $\mathrm{N}$ por volatilização de amônia decorrentes da utilização de ureia não tratada com NBPT totalizaram 19,7 $\mathrm{kg} \mathrm{ha}^{-1}$ de $\mathrm{N}$, em solo úmido, e 13,5kg ha ${ }^{-1}$ de $\mathrm{N}$, em solo saturado (Figura 1A), correspondendo, 


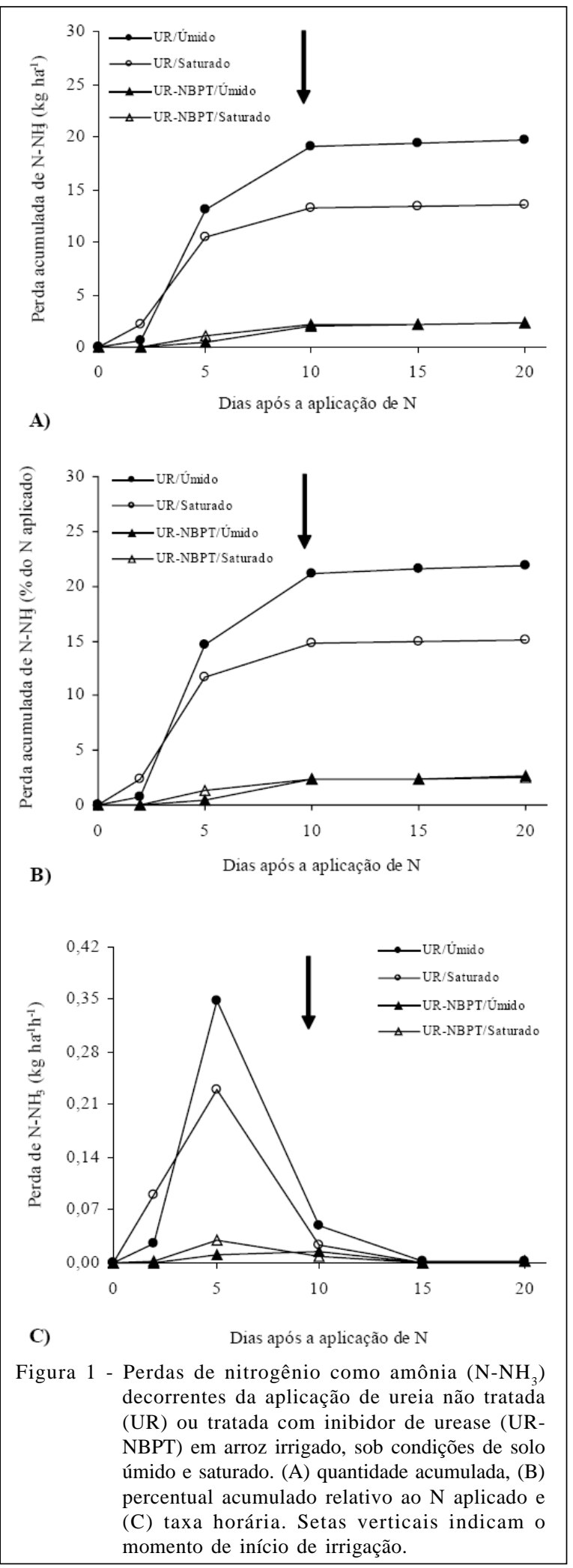

respectivamente, a 21,9 e 15\% do N aplicado (Figura 1B). Provavelmente, as maiores perdas de amônia em solo úmido, em comparação ao saturado, ocorreram em razão da maior atividade da urease naquela condição (CAI et al., 1989), acelerando a hidrólise enzimática da ureia, com a liberação de amônia e dióxido de carbono para a atmosfera. Segundo ZANTUA \& BREMNER (1977), os solos diferem entre si quanto ao nível de atividade da urease, que é determinado por sua habilidade em preservar a ureia da degradação microbiana e de outros processos inativadores. Vários fatores influenciam a atividade da urease no solo, especialmente a temperatura (PETTIT et al., 1976), o pH (DELAUNE \& PATRICK JR., 1970), o conteúdo de água (SANKHAYAN \& SHUKLA, 1976) e a concentração de ureia (AYANABA \& KANG, 1976).

De forma geral, as perdas de nitrogênio por volatilização de amônia decorrem de adubações que promovem elevadas concentrações de $\mathrm{N}$ amoniacal na camada superficial do solo, particularmente em razão da ausência ou incorporação ineficiente do fertilizante (PHONGPAN et al., 1995), sendo mais intensas quando doses elevadas do nutriente são aplicadas nos estádios iniciais de desenvolvimento da cultura (VLEK et al., 1980), condição que está se intensificando nos cultivos de arroz irrigado no Rio Grande do Sul.

Em ambas as condições de umidade do solo, a associação da ureia ao inibidor de urease NBPT reduziu significativamente as perdas de $\mathrm{N}-\mathrm{NH}_{3}$. Os percentuais de redução foram de 88 e $83 \%$, em solo úmido e saturado, respectivamente. No solo úmido, as perdas acumuladas foram de $2,4 \mathrm{~kg} \mathrm{ha}^{-1}$ de $\mathrm{N}(2,6 \%$ do $\mathrm{N}$ aplicado) e, no saturado, de $2,3 \mathrm{~kg} \mathrm{ha}^{-1}$ de $\mathrm{N}$ (2,5\% do $\mathrm{N}$ aplicado) (Figura $1 \mathrm{~A}$ e $1 \mathrm{~B})$. Esses resultados mostram que a ação do NBPT não sofreu influência da umidade do solo. Além disso, indicam que, mesmo no solo saturado (umidade $\cong 24 \%$ ), o NBPT foi convertido a sua forma oxigênio análoga [N-(n-butil) triamida fosfórica] (NBPTO), a qual é efetivamente ativa, retardando a hidrólise da ureia (CREASON et al., 1990; QUI-XIANG et al., 1994). A eficiência do NBPT em solos drenados foi determinada para diferentes culturas (BREMNER \& CHAI, 1986; CARMONA et al., 1990; WATSON et al., 1994; ANTISARI et al., 1996; CANTARELLA et al., 2008), podendo, pelos resultados obtidos neste estudo, ser estendida para o arroz irrigado estabelecido no sistema de semeadura em solo seco, que predomina no Rio Grande do Sul. Porém, sua aplicação em solos inundados, diretamente sobre a lâmina de água, ainda requer avaliação criteriosa, uma vez que o efeito do produto varia com a concentração utilizada, a atividade da urease no solo e sua capacidade em convertê-lo à

Ciência Rural, v.40, n.6, jun, 2010. 
forma oxigênio análoga (QUI-XIANG et al., 1994), os atributos de solo (WATSON et al., 1994), a temperatura (BREMNER \& CHAI, 1986), as práticas de manejo e o crescimento de algas fotossintetizantes (CAI et al., 1989).

$\mathrm{O}$ padrão temporal de perdas de $\mathrm{N}$ por volatilização de amônia da ureia foi semelhante ao determinado em estudos anteriores (ZHU et al., 1989; FRENEY et al., 1995). As perdas foram pequenas até o segundo dia, aumentando de forma marcante até o quinto dia, decrescendo até praticamente estabilizaremse no décimo dia após a aplicação, coincidindo com o momento de submersão do solo. Em ambas as condições de umidade do solo, a adição do inibidor de urease NBPT reduziu as perdas de $\mathrm{N}-\mathrm{NH}_{3}$ até o décimo dia da aplicação. Depois, as quantidades de amônia volatilizada foram desprezíveis, independentemente de a ureia ter sido ou não tratada com NBPT (Figura 1C).

A produtividade de grãos do arroz foi influenciada pela interação entre o tipo de fertilizante e o intervalo de tempo entre sua aplicação no início do perfilhamento do arroz e a submersão do solo. Não houve efeito significativo da umidade do solo e de suas interações com os demais fatores avaliados. Quando a aplicação de nitrogênio foi realizada 10 dias antes da submersão do solo, a associação da ureia ao inibidor de urease NBPT propiciou maior produtividade de grãos, em comparação à ureia não tratada. Esse efeito não se manifestou para os intervalos de um e cinco dias, em que o desempenho de ambas as fontes foi semelhante. Apenas a ureia não tratada com NBPT sofreu a influência do intervalo de tempo entre a aplicação do fertilizante e o início da irrigação. Para esse tipo de fertilizante, observou-se redução na produtividade de grãos com o aumento do período entre a aplicação do fertilizante e a submersão do solo de cinco para 10 dias (Tabela 1).

Resultados semelhantes foram descritos por NORMAN et al. (2004), que determinaram, em duas safras consecutivas, redução na produtividade do arroz em resposta ao aumento do período entre a aplicação de ureia e a inundação do solo de cinco para 10 dias, o que não ocorreu quando associada ao inibidor NBPT. Entretanto, outros estudos reportam ineficiência do NBPT em promover aumento na produtividade do arroz, particularmente quando aplicado em solo inundado, apesar da comprovada redução nas perdas de amônia (CAI et al., 1989; FRENEY et al., 1995; PHONGPAN et al., 1995), sugerindo que o nitrogênio retido no solo, devido ao uso desse inibidor, pode ser convertido à forma nítrica, estando sujeito a perdas por desnitrificação (CAI et al., 1989).
Tabela 1 - Produtividade de grãos e nitrogênio acumulado na parte aérea das plantas de arroz em função do tratamento da ureia com o inibidor de urease NBPT e do intervalo de tempo entre a aplicação de $\mathrm{N}$ e a entrada de água na lavoura.

\begin{tabular}{|c|c|c|c|}
\hline \multirow{2}{*}{ Fonte de $\mathrm{N}$} & \multicolumn{3}{|c|}{--------------Intervalo de tempo-------------' } \\
\hline & 10 dias & 5 dias & 1 dia \\
\hline \multicolumn{4}{|c|}{---------------Produtividade de grãos $\left(\mathrm{kg} \mathrm{ha}^{-1}\right)^{1}$-------------- } \\
\hline Ureia & 7984b B & 8752a A & 9101a A \\
\hline Ureia+NBPT & 8701a A & 8868a A & 9207a A \\
\hline \multicolumn{4}{|c|}{--------Nitrogênio acumulado na parte aérea $\left(\mathrm{kg} \mathrm{ha}^{-1}\right)^{2}$------- } \\
\hline Ureia & $132,0 \mathrm{~b} \mathrm{~B}$ & $149,2 \mathrm{a} A B$ & $162,8 \mathrm{a} A$ \\
\hline Ureia+NBPT & $153,7 \mathrm{a} A$ & $158,6 a \mathrm{~A}$ & $165,1 \mathrm{a} A$ \\
\hline
\end{tabular}

Médias seguidas de mesma letra, minúscula nas colunas e maiúscula nas linhas, não diferem significativamente entre si pelo teste de Tukey $(\mathrm{P}<0,05)$. ${ }^{1}$ coeficiente de variação=9,5\%. ${ }^{2}$ coeficiente de variação=14,8\%.

Acompanhando o efeito observado para a produtividade de grãos, a acumulação de $\mathrm{N}$ na parte aérea das plantas de arroz foi influenciada pela interação entre a fonte de nitrogênio e o período de tempo entre a aplicação do fertilizante e o início da irrigação. Apenas quando a cobertura com $\mathrm{N}$ foi realizada 10 dias antes do alagamento do solo, o uso de NBPT resultou em maior quantidade de $\mathrm{N}$ acumulado. Para os intervalos de tempo menores, não houve diferença significativa entre o tratamento ou não com NBPT. O efeito do período de tempo entre a aplicação de $\mathrm{N}$ e a inundação do solo manifestou-se, apenas, para a ureia não tratada com NBPT. Para esse tipo de fertilizante, maior acumulação de $\mathrm{N}$ foi determinada para o intervalo de um dia, seguido pelo intervalo de cinco dias, com desempenho intermediário, e de 10 dias, que proporcionou menor acumulação de $\mathrm{N}$ (Tabela 1). Esses resultados refletem as perdas de $\mathrm{N}-\mathrm{NH}_{3}$ decorrentes do uso de ureia tratada ou não com o inibidor de urease NBPT no sistema em estudo, confirmando resultados obtidos previamente (FRENEY et al., 1995).

Apesar da aceleração da degradação enzimática da ureia em solo úmido, em comparação ao saturado, condicionando maiores perdas de $\mathrm{N}-\mathrm{NH}_{3}$ (Figura 1), não se verificou influência da umidade do solo por ocasião da primeira aplicação de nitrogênio em cobertura sobre o acúmulo de $\mathrm{N}$ pelo arroz, reforçando suposição de que o $\mathrm{N}$ não volatilizado pode não ser integralmente aproveitado pelas plantas, em razão da perda por outras vias, como a desnitrificação. 


\section{CONCLUSÃO}

As perdas de $\mathrm{N}$ por volatilização de amônia decorrentes do uso de ureia variaram de 15 (solo saturado) a $22 \%$ (solo úmido) do nitrogênio aplicado, quando o intervalo entre a aplicação desse fertilizante e o início da irrigação foi de 10 dias. Com a adição de NBPT, estas foram reduzidas em 83 e $88 \%$, para as condições de solo saturado e úmido, respectivamente.

$\mathrm{O}$ uso de NBPT proporcionou maiores produtividade de grãos e acumulação de nitrogênio pelo arroz apenas quando a aplicação de $\mathrm{N}$ em cobertura antecedeu em 10 dias o início da irrigação.

O tratamento da ureia com NBPT possibilita que sua aplicação anteceda em até 10 dias o início da irrigação, sem prejuízos para a produtividade de grãos e a acumulação de nitrogênio pelo arroz.

\section{REFERÊNCIAS}

AGROTAIN. Agrotain: product information guidebook. Saint Louis, 2001. 55p.

ANJOS, J.T.; TEDESCO, M.J. Volatilização de amônia proveniente de dois fertilizantes nitrogenados aplicados em solos cultivados. Científica, Jaboticabal, v.4, p.49-55, 1976.

ANTISARI, L.V. et al. Effects of the urease inhibitor N-(nbutyl) phosphorothioic triamide in low concentrations on ammonia volatilization and evolution of mineral nitrogen. Biology and Fertility Soils, Berlin, v.22, p.196-201, 1996. Disponível em: <https://springerlink.metapress.com/content/ q 2 n 337 n 56 h 8 u $2070 /$ resource - secured $/$ ? t a r g e t $=\mathrm{f} \mathrm{ull} \mathrm{text}$. pdf \& s id $=\mathrm{z}$ o z c 4 e 45 umyl1145e35t4b32\&sh=www.springerlink.com>. Acesso em: 13 maio, 2010. doi: 10.1007/BF00382512.

AYANABA, A.; KANG, B.T. Urea transformations in some tropical soils. Soil Biology and Biochemistry, Oxford, v.8, p.313-316, 1976. Disponível em: <http://www.sciencedirect.com/ science?_ob=ArticleURL\&_udi=B6TC7-476F84G$3 \mathrm{~F} \&$ u s e r $=686487 \&$ c over D a t e $=12 \%$ 2F31\%2F1976\&_rdoc=12\&_fmt=high\&_orig=browse\&_srch=docinfo(\%23t oc\%235163\%231976\%23999919995\%2 $3360424 \% 23$ F L P \% 23 dis p la y \% 23 Volume ) \&_cdi $=5163 \&$ \& sort $=\mathrm{d} \&$ _docanchor $=\& \mathrm{view}=\mathrm{c} \& \_\mathrm{ct}=16 \&$ _acct $=$ C $000037759 \&$ \& version $=1 \&$ _urlVersion $=$ 0\&_userid $=686487 \& m d 5=8 f 10 \mathrm{da} 9724 \mathrm{c} 4 \mathrm{~d} 4490 \mathrm{f} 28751 \mathrm{~d} 508 \mathrm{a} 7 \mathrm{eb} 8>$. Acesso em: 13 maio, 2010. doi: 10.1016/0038-0717(76)90062-6.

BREMNER, J.M.; CHAI, H.S. Evaluation of N-butyl phosphorothioic triamide for retardation of urea hydrolysis in soil. Communication in Soil Science and Plant Analysis, New York, v.17, n.3, p.337-351, 1986. Disponível em: <http:/ / w w w. inform a world.com/ s m p p/ ftinterface $\sim$ content $=$ a905340998 fulltext $=713240930 \sim$ frm $=$ content $>$. Acesso em: 13 maio, 2010. doi: 10.1080/00103628609367716.

CAI, G.X. et al. The evaluation of urease inhibitors to improve the efficiency of urea as N-source for flooded rice. Soil Biology and Biochemistry, Oxford, v.21, n.1, p.137-145, 1989. Disponível em: $<$ http://www.sciencedirect.com/science?_ob=ArticleURL\&_udi=B6TC747 DKHWR-2X\&_user $=686487 \&$ _coverDate $=12 \% 2 \mathrm{~F}$ $31 \% 2 F 1989 \& \_r d o c=23 \&$ fmt $=$ high\&_orig $=$ browse\&_srch $=$ docinfo ( \% 2 3 toc \% $235163 \% 231989 \% 23999789998$
\%23369591\%23FLP\%23 display\%23Volume)\& _cdi=5163\&_sort =d\&_docanchor=\&view $=c \& \_c t=32 \& \&_{-}$acct $=C 000037759 \&$ C version $=1$ $\&_{-}$url version $=0 \&$ _ userid $=686487 \& \mathrm{~m}$ $\mathrm{d} 5=08 \mathrm{f} 6 \mathrm{~d} 09 \mathrm{ab} 77 \mathrm{~b} 20 \mathrm{c} 57212 \mathrm{e} 3 \mathrm{df3} 3 \mathrm{be} 55826>$. Acesso em: 13 maio, 2010. doi: 10.1016/0038-0717(89)90023-0.

CANTARELLA, $\mathrm{H}$. et al. Fruit yield of Valencia sweet orange fertilized with different $\mathrm{N}$ sources and the loss of applied $\mathrm{N}$. Nutrient Cycling in Agroecosystems, Dordrecht, v.67, p.215223, 2003. Disponível em: <http://www.springerlink.com/ content/h36716770r596446/fulltext.pdf>. Acesso em: 13 maio, 2010. doi: 10.1023/B:FRES.0000003600.20499.76.

CANTARELLA, H. et al. Ammonia volatilization from urease inhibitor-treated urea applied to sugarcane trash blankets. Scientia Agricola, Piracicaba, v.65, p.397-401, 2008. Disponível em: <http://www.scielo.br/pdf/sa/v65n4/11.pdf>. Acesso em: 13 maio, 2010. doi: 10.1590/S010390162008000400011.

CARMONA, G. et al. Temperature and low concentration effects of the urease inhibitor N-(n-butyl) thiophosphoric triamide (nNBPT) on ammonia volatilization from urea. Soil Biology and Biochemistry, Oxford, v. 22, p. 933-937, 1990. Disponível em: $<$ h t t p : / / w w w. sc i e n c ed i r e c t. com/ science? ob=ArticleURL\&_udi=B6TC7-47DKNKTHV\&_user=686487\&_coverDate=12\%2F31\%2F1990\&_rdoc=1\&_fmt=high \&_orig=search\&_sort $=$ d\&_docanchor $=\&$ view $=$ c \&_searc hStrId $=1334588099 \&$ \&erunOrigin $=$ google $\&$ _acct $=$ C00 $0037759 \&$ _ version $=1 \&$ _ url Version $=0 \&$ _u s erid $=686487 \&$ md $5=$ ed50d68d69fa $457 \mathrm{dd} 27467293 \mathrm{~cd} 86 \mathrm{f} 9 \mathrm{~b}>$. Acesso em: 13 maio, 2010. doi: 10.1016/0038-0717(90)90132-J.

COMISSÃO DE QUÍMICA E FERTILIDADE DO SOLO (CQFS RS/SC). Manual de adubação e de calagem para os Estados do Rio Grande do Sul e de Santa Catarina. 10.ed. Porto Alegre: Sociedade Brasileira de Ciência do Solo/Núcleo Regional Sul, 2004. 400p.

COUNCE, P.A. et al. A uniform, objective, and adaptative system for expressing rice development. Crop Science, Madison, v.40, p.436-443, 2000. Disponível em: <http:// crop.scijournals.org/cgi/reprint/40/2/436>. Acesso em: 13 maio, 2010.

CREASON, G.L. et al. Urease inhibitory associated with N-(nbutyl)thiophosphophoric triamide is due to formation of its oxon analog. Soil Biology and Biochemistry, Oxford, v.22, n.2, p.209-211, 1990.

DELAUNE, R.D.; PATRICK JR., W.H. Urea conversion to ammonia in waterlogged soils. Soil Science Society of America Proceedings, Madison, v.34, p.603-607, 1970. Disponível em: <http://soil.scijournals.org/cgi/content/abstract/ 34/4/603>. Acesso em: 13 maio. 2010.

FILLERY, I.R.P. et al. Influence of field environment and fertilizer management on ammonia loss from flooded rice. Soil Science Society of America Journal, Madison, v.48, p.914920, 1984. Disponível em: <http://soil.scijournals.org/cgi/ reprint/48/4/914>. Acesso em: 13 maio. 2010.

FRENEY, J.R. et al. Effect of urease, nitrification and algal inhibitors on ammonia loss and graisn yield of flooded rice in Thailand. Fertilizer Research, The Hague, v.40, p.225233, 1995. Disponível em: <https://springerlink.metapress.com/ content/nu11r6735q5n218u/resource-secured/ ? t arget $=$ fulltext.pdf \& sid =ufip 1045 q q u4gq455qugz1fr\&sh=www.springerlink.com>. Acesso em: 13 maio, 2010. doi: 10.1007/BF00750469. 
LARA CABEZAS, W.A.R. et al. Volatilização de N-NH3 na cultura do milho: I. Efeito da irrigação e substituição parcial de uréia por sulfato de amônio. Revista Brasileira de Ciência do Solo, Campinas, v.21, p.481-487, 1997. Disponível em: $<$ http://sbcs.solos.ufv.br/solos/revistas/v21n3a18.pdf $>$. Acesso em: 13 maio, 2010

LARA CABEZAS, W.A.R. et al. Balanço da adubação nitrogenada sólida e fluida de cobertura na cultura de milho, em sistema plantio direto no triângulo mineiro (MG). Revista Brasileira de Ciência do Solo, Viçosa, v.24, p.363-376, 2000. Disponível em: <http://sbcs.solos.ufv.br/solos/revistas/ v24n2a14.pdf $>$. Acesso em: 13 maio, 2010

NÖMMIK, H. The effect of pellet size on the ammonia loss from urea applied to forest soil. Plant and Soil, The Hague, v.39, p.309-318, 1973. Disponível em: <https:// springerlink.metapress.com/content/u833m82hw2p271t1/ resource-secured/? target=fulltext.pdf \& sid=ufip 104 5qqu4gq455qugz1fr\&sh=www.springerlink.com>. Acesso em: 13 maio,2010. doi: 10.1007/BF00014798.

NORMAN, R.J. et al. Fate of different $\mathrm{N}$ fertilizers applied preflood to drill-seeded, delayed flood rice. In: ANNUAL NATIONAL CONSERVATION TILLAGE COTTON \& RICE CONFERENCE, 7., 2004, Little Rock, AR. Proceedings... Perryville: National Conservation Tillage Digest, 2004. p.3335.

PETTIT, N.M. et al. Soil urease: activity stability and kinetic properties. Soil Biology and Biochemistry, Oxford, v.8, p.479-484, 1976. Disponível em: <http://www.sciencedirect.com/ science? ob=MImg\&_imagekey=B6TC7-47DKSNF-Y $1 \&$ \&_di $=5163 \&$ \&_user $=10 \&$ \& pii $=003807177690$ $0894 \&$ orig=search \& coverDate $=12 \% 2 \mathrm{~F} 31 \% 2 \mathrm{~F} 19$ $76 \&$ \& s $=999919993 \&$ vi ew $=$ c \& w chp $=$ d G L b VzW zSkWb\&md5=59abad7b 574bda1 8ccecad3d41185759\&ie=/ sdarticle.pdf $>$. Acesso em: 13 maio, 2010. doi:10.1016/00380717(76)90089-4.

PHONGPAN, S. et al. Use of phenylphosphorodiamidate and $\mathrm{N}$-(n-butyl)thiophosphorictriamide to reduce ammonia loss and increase grain yield following application of urea to flooded rice. Fertilizer Research, The Hague, v.41, p.59-66, 1995. Disponível em: <https://springerlink.metapress.com/content/ n 0106 h 829165 t 228 /res ource-secured/? $\mathrm{t}$ a r g e t $=\mathrm{f} \mathrm{ull} \mathrm{text}$. pd f \& s i d = uf i p $1045 \mathrm{qqu} 4 \mathrm{gq}$ 455qugz1fr\&sh=www.springerlink.com>. Acesso em: 13 maio, 2010. doi: 10.1007/BF00749521.

QUI-XIANG L. et al. Inhibition of urease activity in flooded soils by phenylphosphorodiamidate and $\mathrm{N}$-(n-butyl)thiophosphorictriamide. Soil Biology and Biochemistry, Oxford, v.26, n.8, p.1059-1065, 1994. Disponível em: <http://www.sciencedirect.com/ science?_ob=MImg\&_imagekey=B6TC7-476MVGN-92 $1 \&$ c d i $=5163 \&$ \& s e $r=10 \&$ p i i $=0038$ $07179490121 \mathrm{X} \&$ \& orig=article \&_coverDate $=$ 08\%2F31\%2F1994\& sk=999739991\&view $=c \& w c h p=d G L b V t z-$ zSkWb\&md5 = f0abd6fd3d038dab c957b74f6b8ae842\&ie =/ sdarticle.pdf $>$. Acesso em: 13 maio, 2010. doi:10.1016/00380717(94)90121-X

SANKHAYAN, S.D.; SHUKLA, V.C. Rates of urea hydrolysis in five soils of India. Geoderma, Amsterdam, v.16, p.171178, 1976. Disponível em: <http://www.sciencedirect.com/ science?_ob=MImg\&_imagekey=B6V67-48BCGBX-4D$1 \&$ c d i $=5807 \&$ u s e $r=10 \& \quad$ p i i $=00167$ $06176900380 \&$ orig = search \& coverDate $=09 \%$ 2F30\%2F1976\&_sk=999839997\&view =c\&wchp=dGLzVtzzSkzS\&md5=92a980f5e 5ba8dba 481 8309274e87aa1\&ie=/ sdarticle.pdf $>$. Acesso em: 13 maio, 2010. doi: 10.1016/00167061(76)90038-0
SOCIEDADE SUL-BRASILEIRA DE ARROZ IRRIGADO (SOSBAI). Arroz irrigado: recomendações técnicas da pesquisa para o Sul do Brasil. Pelotas: SOSBAI, 2007. 154p.

VENTURA, W; YOSHIDA, T. Ammonia volatilization from a flooded tropical soil. Plant and Soil, The Hague, v.46, p.521531, 1977. Disponível em: <https://springerlink.metapress.com/ content/tl 70 h 8250137 rj0x/resource-secured/ ? t a r g e $t=f u l l t e x t$. pd f \& s i d $=u f i$ p1045qqu4gq455qugz1fr\&sh=www.springerlink.com>. Acesso em: 13 maio, 2010. doi:10.1007/BF00015911.

VITTI, A.C. et al. Produtividade da cana-de-açúcar relacionada à localização de adubos nitrogenados aplicados sobre os resíduos culturais em canavial sem queima. Revista Brasileira de Ciência do Solo, Viçosa, v.31, p.491-498, 2007. Disponível em: <http:/ /www.scielo.br/scielo.php?pid=S0100-06832007000300009 \&script $=$ sci _abstract\&tlng=pt $>$. Acesso em: 13 maio, 2010. doi: 10.1590/S0100-06832007000300009.

VLEK, P.L.G. et al. Urease activity and inhibition in flooded systems. Fertilizer Research, The Hague, v.1, p.191-202, 1980. Disponível em: <https://springerlink.metapress.com/ content/j20451u4764430 g 3/resource-secured/ ? t a r get $=$ f u ll text.pdf \& si d = uf i p 1045 q qu 4 gq455qugz1fr\&sh=www.springerlink.com>. Acesso em: 13 maio, 2010. doi: 10.1007/BF01053131.

WATSON, C.J. et al. Agronomic assessment and ${ }^{15} \mathrm{~N}$ recovery of urea amended with the urease inhibitor nBTPT (N-(n-butyl) thiophosphoric triamide) for temperate grassland. Plant and Soil, The Hague, v.161, p.167-177, 1994. Disponível em: <https:/ /springerlink.metapress.com/content/q7036p1l3662n767/ resource-secured/?target=fulltext.pdf\&sid=ufip $1045 q \mathrm{qq}$ u4gq455qugz1fr\&sh=www.springerlink.com>. Acesso em: 13 maio, 2010. doi: 10.1007/BF00046388.

XU, J.G. et al. Fertilizer and temperature effects on urea hydrolysis in undisturbed soil. Biology and Fertility of Soils, Berlin, v.16, p.63-65, 1993. Disponível em: <https:// springerlink.metapress.com/content/p91rrx0243577643/ resource-secured $/$ ? target $=$ fulltext.pdf \& sid=ufip 104 5qqu4gq455qugz1fr\&sh=www.springerlink.com>. Acesso em: 13 maio, 2010. doi: 10.1007/BF00336517.

ZANTUA, M.I.; BREMNER, J.M. Stability of urease in soils. Soil Biology and Biochemistry, Oxford, v.9, p.135140, 1977. Disponível em: <http://www.sciencedirect.com/ science?_ob=MImg\&_imagekey=B6TC7-476NBD2-PJ$1 \&$ \&_di $=5163 \&$ \&_user $=10 \&$ \&_pi $=003807177790050$ $5 \&$ \& orig=browse \&_coverDate $=12 \% 2$ F $31 \% 2$ F $1977 \&$ $\mathrm{sk}=999909997 \& \mathrm{view}=\mathrm{c} \& \mathrm{w} \mathrm{chp}=\mathrm{dGLzVzzzSkzS}$ $\& m d 5=78147$ b 0 c ac $09 d 4$ a 8 c 720 eeb $2 b 17 \mathrm{ffaa} 8 \& i e=/$ sdarticle.pdf $>$. Acesso em: 13 maio, 2010. doi: 10.1016/ 0038-0717(77)90050-5

ZHU, S.L. Efficient management of nitrogen fertilizers for flooded rice in relation to nitrogen transformations in flooded soils. Pedosphere, Nanjing, v.2, p.97-114, 1992.

ZHU, Z.L. et al. Processes of nitrogen loss from fertilizers applied to flooded rice fields on calcareous soil in north-central China. Fertilizer Research, The Hague, v.18, p.101-115, 1989. Disponível em: <https://springerlink.metapress.com/ content/j195l632q8645672/resource-secured/ ? t a r g e $\mathrm{t}=\mathrm{f}$ u l l t e $\mathrm{xt}$. p d f \& s i d = u f i p 1045 qqu4gq455qugz1fr\&sh=www.springerlink.com>. Acesso em: 13 maio, 2010. doi: 10.1007/BF01049507. 